\title{
Temor a la evaluación social negativa: validez de constructo y criterio del instrumento de medición*
}

\author{
Fear of Negative Social Evaluation: Construct and \\ Criterion Validity of the Measurement Instrument
}

ecibido: mayo 3 de 2011 | Revisado: mayo 15 de 2012 | Aceptado: agosto 15 de 2012

\author{
Christian Enrique Cruz-Torres ** \\ ROLANDO DíAZ-LOVING *** \\ REBECA EsTHER OROPEZA-LOZANO ***** \\ PaOla Eunice DÍAz-Rivera ****** \\ JOAQuín AlberTo PADILla-BAUTISTA ******* \\ ILSE GONZÁLEZ-RIVERA ******** \\ Universidad Nacional Autónoma de México, México
}

doi:10.11144/Javeriana.UPSY12-2.tesn

Para citar este artículo: Cruz, C., E., Díaz-Loving, R., Oropeza, R. E., Díaz, P. E., Padilla, J. A. \& González, I. (2013). Temor a la evaluación social negativa: validez de constructo y criterio del instrumento de medición. Universitas Psychologica, 12(2), 531-545.

* Este estudio forma parte de una investigación más amplia, apoyada por todos los integrantes de la Unidad de Investigaciones Psicosociales, en la Facultad de Psicología de la Universidad Nacional Autónoma de México. No recibió apoyo financiero directo de otras instituciones educativas, del Gobierno ni de empresas privadas.

** Universidad Nacional Autónoma de México. E-mail: cassiel.79@gmail.com

**** Profesor Investigador en la Facultad de Psicología de la Universidad Nacional Autónoma de México. Director de la Unidad de Investigaciones Psicosociales.E-mail: rdiazl@unam.mx

****** Investigadora en la Unidad de Investigaciones Psicosociales.E-mail: rbkesther@yahoo.com.mx

******* Universidad Nacional Autónoma de México e investigadora en la Unidad de Investigaciones Psicosociales.E-mail: flordenovalis@gmail.com

${ }^{* * * * * * * * *}$ Investigador becario de la Unidad de Investigaciones Psicosociales, Universidad Nacional Autónoma de México.E-mail: nembrod@hotmail. com

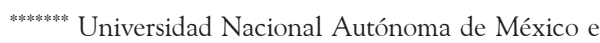
investigadora en la Unidad de Investigaciones Psicosociales. E-mail: ilse1205@hotmail.com

\section{RES U MEN}

El Instrumento de Temor a la Evaluación Social Negativa (ITESN) ha mostrado evidencias de validez y confiabilidad al utilizarse en muestras culturalmente distintas y al traducirse a diferentes idiomas (Duke et al., 2006; Gallego, 2010; Gallego et al., 2007; Weeks et al., 2005; Tavoli et al., 2009). Dada la relevancia del estudio de la ansiedad y fobia social, y con la intención de aportar herramientas para estudiar estas variables en muestras mexicanas, este proyecto presenta evidencias de validez y confiabilidad de una traducción al castellano del ITESN (Leary, 1983) en una muestra de estudiantes universitarios de Ciudad de México. Se realizaron análisis de discriminación de reactivos, factoriales exploratorios y factoriales confirmatorios mediante modelamiento de ecuaciones estructurales. Los resultados muestran una estructura unifactorial que agrupa ocho de los doce reactivos del instrumento original, explica el $53.8 \%$ de la varianza total y muestra consistencia interna adecuada $(\alpha=0.88)$. Se obtienen evidencias de validez de criterio del ITESN, así: a) se asocia positivamente con el Instrumento de Ansiedad Rasgo y b) mediante un procedimiento experimental, se observan asociaciones positivas entre el ITESN y mediciones de ansiedad estado y motivación de autopresentación ante la expectativa de conocer a otra persona. Se discute el funcionamiento de los cuatro reactivos redactados de forma negativa y la interpretación teórica de las relaciones que respaldan la validez de criterio.

Palabras clave autores

Ansiedad social, psicometría, validación experimental.

Palabras clave descriptores

Evaluación social, interacción, ansiedad social, autopresentación, validación.

\begin{abstract}
A B S T R A C T
The Brief fear of Negative Evaluation Scale (BFNE) has shown good validity and reliability properties in different cultural samples, as well as when it has been translated to other languages (Duke et al., 2006; Gallego, 2010; Gallego et al., 2007; Weeks et al., 2005; Tavoli et al., 2009). Given the relevance of the study of anxiety and social phobia and the need for research tools for Mexican samples, this study presents validity and reliability evidences for a translation to Spanish language of the BFNE (Leary, 1983) in a sample
\end{abstract}


of undergraduate students from Mexico City. Psychometric tests included discrimination analysis for each item, principal component factorial analysis, and confirmatory factor analysis through structural equation modeling. Results show a one factor structure with just eight of the 12 original items. It explains $53.8 \%$ of total variance and has good reliability ( $\alpha=0.88$ ). In addition, evidence was found concerning criterion validity for the BFNE: a) It correlates positively with a Trait Anxiety Scale; and b) through an experimental procedure, positive correlations for the BFNE with a State Anxiety Scale and a Self-Presentation Motivation Scale when participants had the expectation of being introduced to another person. The discussion verses in relation to the performance of score reversed items and the theoretical meaning of items and to the observed relationships that support the criterion validity of this translation to Spanish language of the BFNE scale.

Key words authors

Social Anxiety, Psychometry, Experimental Validation.

Key words plus

Social Evaluation, Interaction, Social Anxiety, Self-presentation, Validation.

\section{Introducción}

Los seres humanos, en tanto seres sociales, están diseñados para y tienen la necesidad de vivir en grupos con otros seres humanos; por esto, es relevante para los individuos la forma en que son evaluados por sus pares y evitar el rechazo o la exclusión definitiva del grupo (Díaz-Loving \& Draguns, 1999). De esta forma, la evaluación social -es decir, cómo valoran los otros al individuo- es una manera en que las personas aprenden sobre sí mismas, hacen mejoras y buscan información relevante para poder adaptarse dentro de su medio (Gómez-Jacinto, 2005).

Una de las formas en que el individuo percibe esta evaluación social es mediante la comparación con otros. La teoría de la comparación social (Festinger, 1954) indica que el individuo se compara con otros al encontrarse inseguro sobre sus habilidades u opiniones, principalmente cuando se carece de estándares físicos u objetivos.

Esta comparación es una necesidad biológica y continua de los seres humanos que ubica al individuo en la jerarquía de su grupo, evitando confrontaciones peligrosas mediante el establecimiento del estatus (Bunnk \& Mussweiler, 2001; Gartrell, 1987; Gómez-Jacinto, 2005). Además, este proceso de comparación social influye en la autoestima, la autoevaluación y el autoconcepto del individuo, calibrando el grado de aceptación grupal (Bunnk \& Mussweiler, 2001; Festinger, 1954; Gómez-Jacinto, 2005).

El grado de aceptación social tiene una repercusión directa a nivel cognoscitivo, emocional y conductual en el individuo. Por ejemplo, se sabe que los niños y adolescentes que son aceptados por sus iguales reciben más reforzamiento social, mejorando su adaptación en áreas sociales y personales (Inderbitzen, Walters \& Bukowski, 1997; La Greca \& López, 1998). Otros estudios muestran que una menor aceptación y valoración de los pares ocasiona que se muestren menos seguros de su competencia social en interacciones sociales (Patterson, Kupersmidt \& Griesler, 1990; Sánchez \& Díaz-Loving, 2009).

La evaluación que los otros relevantes hacen de nuestro comportamiento es tan significativa que modelos como la teoría de la conducta planeada (Ajzen, 1991) y la teoría de la conducta interpersonal (Triandis, 1977) la incluyen en sus modelos como un factor determinante de la toma de decisiones para llevar a cabo o no una cierta conducta.

El ser humano experimenta una tendencia natural a establecer relaciones interpersonales (Leary, 1983; Zubeidat, 2005) que le demandan una modificación constante de su comportamiento, derivando en diferentes niveles de ansiedad. Esta es una emoción adaptativa y necesaria que, entre otras cosas, motiva a los seres humanos a actuar adecuadamente ante situaciones interpersonales (Botella, Baños \& Perpiñá, 2003; Díaz-Loving, 2002).

Dicha ansiedad surge en respuesta a exigencias, amenazas o problemas sociales que deben resolverse de forma independiente (Hernández, Orellana, Kimelman, Núñez \& Ibañez, 2005; Hidalgo \& Abarca, 1990) para adaptarse, aunque disminuye tras los primeros momentos de interacción social y al exponerse repetidamente a situaciones parecidas. Sin embargo, cuando la ansiedad se presenta de manera excesiva y constante ante acciones como el beber, comer o hablar ante los demás, se habla de un trastorno de ansiedad. Este se define como una 
experiencia de estrés, incomodidad y miedo ante situaciones sociales, ante la posibilidad de hacer el ridículo o ser evaluado negativamente por los demás, impidiendo que el sujeto interactúe y sostenga relaciones interpersonales adecuadamente (American Psychiatric Association, 2000; Bados, 2009; Duke, Krishnan, Faith \& Storch, 2006; Watson \& Friend, 1969 citado en Zubeidat, Fernández-Parra \& Sierra, 2006; Zubeidat, Salinas \& Sierra, 2007).

Este temor a la evaluación social negativa puede variar en intensidad, siendo en un extremo tan bajo que algunas personas se sienten muy seguras de su desempeño ante los demás e ignoran la evaluación que hacen de ella. En el otro extremo el miedo a esta evaluación puede ser tan alto que impide llevar a cabo tareas de forma eficiente, diagnosticándose como un trastorno de ansiedad social. Este trastorno se refleja en diferentes niveles de respuesta: el fisiológico, donde la persona puede presentar malestar gástrico, sudoración, temblor de voz; el conductual, que puede manifestarse en dificultades para expresarse, evitar ser el centro de atención o evitar interactuar con los demás; y el cognoscitivo, donde surgen dificultades para recordar cosas importantes o para concentrarse (Gallego, 2010).

Watson y Friend (1969 citado en Zubeidat et al., 2006) definen el miedo a la evaluación negativa como un temor hacia la valoración de los demás y a las expectativas que se generan alrededor de la misma, donde la desaprobación causa un malestar exagerado en el individuo, al grado que puede evitar los estímulos que le parecen amenazantes y abandonar sus actividades cotidianas. Sin embargo, los procesos sociales en los que el temor a la evaluación es un factor importante deben ser juzgados según las diferencias en el nivel en que las personas temen a ser evaluados negativamente por otros (Leary, 1983). Es decir, quienes están muy preocupados por ser evaluados negativamente son más propensos a evitar dichas evaluaciones y responder más intensamente ante situaciones que les provocan ansiedad.

El estudio de Kessler et al. (1994 citados en Rapee \& Himberg, 1997), reporta que la ansiedad social es el trastorno más prevalente en E.E. U.U., donde un $13 \%$ de la población es diagnosticada en algún punto de su vida con este trastorno. Actual- mente, se le considera el tercer trastorno psicológico con mayor prevalencia tras la depresión mayor y la dependencia del consumo de alcohol (American Psychiatric Asociación, 2000).

Estos índices resultan relevantes dado el impacto de este trastorno en la vida cotidiana de quienes lo padecen. Beidel y Turner (1998) indican que las consecuencias de este trastorno en el proceso de adaptación del adolescente pueden implicar un bajo rendimiento académico y con ello el riesgo de abandono escolar. Varios estudios dan cuenta del impacto de este trastorno sobre el funcionamiento laboral, lúdico y emocional de la persona (Rapee, 1995; Schneier et al., 1994 citados en Rapee \& Himberg 1997; Zubeidat, 2005). El estudio de García (2000) muestra además que las personas que sufren ansiedad experimentan una menor aceptación y valoración social, se muestran menos atractivos hacia el sexo opuesto y presentan una mayor dificultad en establecer tanto amistades como relaciones de pareja. Es debido la alta prevalencia de este trastorno y a su impacto en la vida cotidiana que las investigaciones en relación con la ansiedad social han ido en aumento en los últimos años (Gallego, 2010).

Crear mejoras para su tratamiento y detección depende de la fiabilidad y validez de los instrumentos empleados en entornos clínicos y de investigación. Por ello, es importante contar con un instrumento que permita medir adecuadamente el temor a la evaluación social negativa en comunidades de habla hispana, dado que es identificado cada vez más como una condición tratable (Duke et al., 2006).

\section{Antecedentes de la medición de Temor a la Evaluación Social Negativa}

En 1969 Watson y Friend crearon el Instrumento de Temor a la Evaluación Social Negativa (ITESN), un instrumento de 30 reactivos en un formato de respuesta verdadero-falso. El ITESN ha resultado útil en investigaciones de personalidad y psicología social, pero ha dejado de utilizarse debido a que su longitud. Ante este problema, Leary (1983) buscó desarrollar una versión breve del ITESN sin sa- 
crificar sus propiedades psicométricas; seleccionó los doce ítems de la escala original que mostraron correlaciones con el total de la escala por arriba de 0.5, modificando además el formato de respuesta de cierto-falso a una escala tipo Likert de 5 puntos.

Esta versión breve presentó una alta correlación con la escala original $(r=0.96, p<0.001)$, adecuados niveles de consistencia interna (alfa de Cronbach $=0.9$ ) y un coeficiente de confiabilidad test-retest de 0.75 que es incluso superior al 0.68 reportado por la escala original de Watson y Friend (1969 citados en Zubeidat et al., 2006). Esta nueva versión del ITESN (Leary, 1983) es usada comúnmente para determinar el grado en que cada persona experimenta aprensión a la posibilidad de ser evaluado negativamente, principalmente en estudios relacionados con la ansiedad social.

Estudios como el de Duke et al. (2006) y Weeks, Heimberg, Fresco, Hart y Turk (2005) han reportado indicadores de validez de constructo de este instrumento. Utilizando la técnica de análisis factorial confirmatorio, ambos estudios ponen a prueba dos modelos teóricos: un modelo que agrupa los 12 reactivos hacia una sola variable latente y un segundo modelo donde los ocho reactivos que denotan un alto temor a la evaluación social negativa se agrupan hacia una variable latente y los cuatro reactivos restantes se agrupan hacia la otra que describe la ausencia de este temor, estando ambas variables latentes correlacionadas negativamente entre sí. Cabe mencionar que ambos estudios llegaron a la misma estructura factorial del instrumento aunque fue aplicado a muestras distintas: una muestra con diagnóstico de desorden de ansiedad social en el caso de Weeks et al. (2005) y una muestra sin diagnóstico en el caso de Duke et al. (2006). Esta misma estructura de dos factores fue replicada también por Tavoli, Melyani, Bakhtiari, Ghaedi y Montazeri (2009) utilizando la técnica de análisis factorial de componentes principales.

Los análisis de consistencia interna han reportado niveles de confiabilidad adecuados para este instrumento. En muestras con un diagnóstico de desorden de ansiedad social, Weeks et al. (2005) reportan un alfa de 0.92 y Tavoli et al. (2009) un alfa de 0.9. Estudios realizados en muestras sin un diagnóstico clínico también han reportado niveles aceptables de confiabilidad. Duke et al. (2006) reportan un alfa de 0.94 para el factor positivo, de 0.73 para el factor inverso y de 0.8 para la totalidad de la escala de 12 reactivos. De forma similar, Weeks et al. (2005) reportan un alfa de 0.9. y Tavoli et al. (2009) de 0.82 para el total de la escala. Además, se han realizado análisis de confiabilidad test-retest para este instrumento, encontrándose coeficientes de correlación de entre 0.71 (Tavoli et al., 2009) y 0.75 (Leary, 1983).

Respecto a la validez de criterio se han encontrado relaciones teóricamente congruentes entre el ITESN y otros constructos relevantes. Se han reportado correlaciones positivas entre este instrumento y la Escala de Interacción Social y Ansiedad (Brown \& Stopa, 2008; Tavoli et al., 2009), el Inventario de Ansiedad Rasgo Estado (Carter, Sbrocco \& Ayati, 2009), la Escala de Fobia Social (Brown \& Stopa, 2008; Tavoliet al., 2009) y el Inventario de Depresión de Beck (Brown \& Stopa, 2008), la Escala de Soledad de la UCLA (Duke et al., 2006) y la Escala de Evitación y Malestar Social (Gallego, 2010). Además se reportan correlaciones negativas con el Cuestionario de Autoeficacia al Intervenir en Público y las dos subescalas del Cuestionario de Autoverbalizaciones durante la Situación de Hablar en Público (Gallego, 2010).

También se ha puesto a prueba la capacidad del instrumento para discriminar entre muestras con y sin un diagnóstico de ansiedad social. Gallego (2010), Tavoli et al. (2009) y Weeks et al. (2005) han mostrado evidencias de esta capacidad del ITESN. Vale la pena mencionar que estos resultados han sido consistentes aún con participantes de diferentes países e idiomas: estadounidenses (Duke et al., 2006; Weeks et al., 2005), iraníes (Tavoli et al., 2009) y españoles (Gallego, 2010; Gallego, Bottella, Quero, Baños \& GarcíaPalacios, 2007). Estos resultados en su conjunto aportan evidencia de que el ITESN es una medida confiable y válida, llevando a la posibilidad de utilizar una traducción al español de este instrumento en proyectos de investigación y, con un trabajo posterior más profundo, en el diagnóstico 
de trastornos de ansiedad en población mexicana o de habla hispana en general.

En vista del buen funcionamiento de este instrumento y de la utilidad que puede reportar al estudio de los trastornos de ansiedad y otros constructos teóricos y problemáticas relacionadas, el presente proyecto tuvo por objetivo obtener indicadores de su validez y consistencia interna en una muestra de participantes mexicanos. Investigaciones previas (Gallego, 2010; Gallego et al., 2007) han reportado indicios del funcionamiento de una traducción al español en una muestra clínica y de estudiantes españoles. El presente proyecto puso a prueba este instrumento en una muestra también de habla hispana aunque culturalmente distinta. Aporta además evidencia de la validez predictiva obtenida desde una aproximación experimental, información no reportada en ninguna de las investigaciones previas. Se espera que el ITESN reporte buenos indicadores de validez de constructo, validez de criterio y consistencia interna. Además, que los puntajes obtenidos se asocien positivamente con una mayor respuesta ansiosa ante la expectativa de interactuar con un desconocido, mostrando así una adecuada capacidad predictiva.

\section{Método}

\section{Participantes}

Participaron 114 hombres y 113 mujeres. La edad promedio fue de 19.7 años $(D E=2.1)$. De los 227 participantes, 95 lo hicieron como parte de los requerimientos de evaluación en materias de los primeros semestres de psicología y los 132 restantes fueron invitados por los mismos estudiantes.

\section{Instrumentos}

Se llevó a cabo una traducción al español del ITESN (Leary, 1983) compuesta de 12 reactivos presentados en formato tipo Likert pictográfico (Reyes, 1993) con siete opciones de respuesta, donde los extremos denotaban un continuo de me describe poco a me describe mucho (ver Apéndice). Cuatro de los doce reactivos tienen una redacción negativa, denotando bajo temor a la evaluación social negativa.

Para obtener evidencias de validez de criterio se utilizó además el Instrumento de Ansiedad RasgoEstado ([IDARE]; Spielberger \& Díaz, 1975) en formato tipo Likert con opciones de respuesta de 1 (casi nunca) a 4 (casi siempre). Este instrumento se compone de dos subescalas. La primera se compone de 20 reactivos y describe los niveles de ansiedad justo en el momento de la evaluación (ansiedad estado). La mitad de los reactivos describen bajos niveles de ansiedad (p. ej., "Me siento calmado") y el resto altos niveles de ansiedad (p. ej., "Me siento ansioso"). La segunda escala se compone de 20 reactivos que describen los niveles de ansiedad como un rasgo estable del participante (ansiedad rasgo). Ocho de estos reactivos denotan bajos niveles de ansiedad (p. ej., "Soy una persona tranquila, serena, sosegada") y el resto altos niveles de ansiedad (p. ej., "Cuando pienso en los asuntos que tengo entre manos me pongo tenso y alterado"). La estructura del IDARE permite, en el contexto de este estudio, evaluar la capacidad del ITESN de medir esta característica como un rasgo estable, además de ser sensible a variaciones temporales en este rasgo como respuesta a estímulos del ambiente. Por esta razón, en el presente proyecto se generan indicadores independientes, uno para el IDAE (Instrumento de Ansiedad Estado) y otro para el IDAR (Instrumento de Ansiedad Rasgo).

Además, se utilizó una medición de motivación de auto presentación (MAP) diseñada ex profeso para este estudio ${ }^{1}$, compuesta de seis reactivos en formato tipo Likert con siete opciones de respuesta. Tres de los reactivos denotaban alta motivación (p. ej., "Es importante para mí generar una buena impresión en la persona que voy a conocer") y los tres restantes baja motivación (p. ej., "No me preocupa lo que la persona que voy a conocer piense de mí”).

1 Dado que la medición de MAP fue diseñada ex profeso para este estudio y considerando que funcionará como criterio externo de validez para la medición de TESN, en la sección de resultados de este mismo artículo se ofrecen algunas evidencias de validez y consistencia interna de este primer instrumento (MAP). 


\section{Materiales}

Cuatro fotografías, dos de hombres y dos de mujeres, previamente evaluadas mediante un procedimiento de jueces con base en su atractivo (Zacarías, 2009), dos eran consideradas claramente más atractivas ${ }^{2}$.

\section{Procedimiento}

Los participantes acudían al laboratorio para programar la hora y la fecha de su participación en el estudio. Elegían entre varios horarios disponibles y se les mencionaba que era importante que llegaran puntuales a su cita. En la historia encubierta utilizada en el procedimiento experimental, se les informaba que conocerían a otro participante invitado a la misma hora que ellos; para dar credibilidad a este punto solo se recibía a los participantes que llegaban con un máximo de 20 minutos después de la hora programada. Quienes llegaban más tarde eran programados para una cita posterior.

Los participantes eran recibidos individualmente en el laboratorio y durante el procedimiento se encontraban solos o acompañados por el experimentador. Al llegar se les pedía que respondieran el ITESN y el IDAR. La información de los procedimientos experimentales siguientes no se les proporcionaba hasta que terminaban de responder estos instrumentos para evitar un sesgo en sus respuestas.

Posteriormente se les explicaba que el estudio era sobre cómo interactuamos con quienes no conocemos, que conocerían a alguien y finalmente responderían algunas preguntas, pero antes sería necesario hacer un intercambio de fotografías para verificar que no se conocían. Después se les

2 En su estudio, Zacarías (2009) lleva a cabo un procedimiento de clasificación de 12 fotografías de hombres y 12 de mujeres con base en su atractivo. Para esto, pide a 200 participantes (estudiantes universitarios) que clasifiquen las fotografías de 12 personas del sexo contrario, de la más a la menos atractiva ("iCon cuál de estas personas te gustaría iniciar una relación de pareja?”). Las fotografías utilizadas en este estudio fueron seleccionadas con base en dos criterios. Primero, que se ubicaran en el extremo de clasificación, una claramente más atractiva que la otra. Segundo, que permitieran hacer creíble la historia encubierta. Es decir, que fueran fotos de personas que parecieran realmente alumnos de la universidad. mostraba la fotografía de quien supuestamente conocerían, quien estaba en ese momento en otro laboratorio. Para controlar un posible sesgo debido al uso de alguna fotografía en particular, se utilizaron de forma aleatoria las cuatro fotografías diferentes.

Después de confirmar que no conocían a la persona en la fotografía, se les pedía que se tomaran una fotografía para mostrarla al otro invitado, informándoles que podían tomarse varias hasta tener una que consideraran adecuada. El experimentador salía del laboratorio, advirtiendo que imprimiría la fotografía para mostrarla al otro participante para garantizar que no se conocieran. Antes de salir, el experimentador pedía al participante que contestara el IDAE y el instrumento de MAP y anunciaba que al regresar irían al otro laboratorio a conocer a la persona de la fotografía.

Al regresar, el experimentador llevaba a cabo la entrevista posexperimental para verificar que el participante creyera que iba a conocer a alguien. Después informaba los verdaderos objetivos y procedimientos del estudio, y pedía no revelar los detalles con otros participantes potenciales.

\section{Resultados}

De los 227 participantes, 15 no creyeron la historia encubierta. En los análisis correspondientes a las primeras dos mediciones (ITESN e IDAR) se utilizaron los datos de los 227 participantes. Para los análisis posteriores, que dependían del éxito de la manipulación experimental, se utilizaron solo los datos de 212 participantes que creyeron la historia encubierta.

\section{Capacidad de discriminación de cada reactivo}

Para obtener indicadores del funcionamiento de cada reactivo del ITESN se conformó una variable, sumando los puntajes del total de los reactivos, y se establecieron grupos de puntajes altos y bajos con base en los cuartiles de esa variable. Después, se compararon los promedios de cada reactivo entre los grupos altos y bajos mediante una prueba $t$ de 
Student ${ }^{3}$. Diferencias estadísticamente significativas entre ambos grupos denotarían que el reactivo puede discriminar a quienes reportan puntajes altos o bajos en el total de la escala. Tres de los 12 reacti$\operatorname{vos}(2,7$ y 10 , denotando bajo temor a la evaluación social negativa $\left.{ }^{4}\right)$ no obtuvieron buenos resultados (todas las $t<0.15$; sig. $>0.116$ ). Estos reactivos no fueron incluidos en los análisis posteriores.

3 Los valores de la prueba de sesgo (entre -0.04 y 0.75 ) y curtosis $(-0.25$ y -1.14$)$ reflejan en general una distribución cercana a la normal para todos los reactivos. La prueba de Levene para igualdad de varianzas muestra varianzas equivalentes entre los grupos de puntajes altos y bajos en 5 de los 12 reactivos. En los reactivos en donde no se puede asumir igualdad de varianzas se utilizaron para el análisis los indicadores ajustados, que para este fin provee el paquete estadístico SPSS. Estas características de los reactivos y procedimientos de análisis, aunados al nivel de medición de los reactivos y la independencia de los puntajes entre ambos grupos permiten suponer que se cumplen los supuestos requeridos para el uso de la prueba $t$.

4 Dado que las comparaciones se hacen reactivo por reactivo, el efecto de la posible diferencia al invertir el sentido de la calificación es exactamente el mismo, aunque con el signo contrario.

\section{Validez de constructo}

Se obtuvieron evidencias de la validez de constructo del instrumento sometiendo los nueve reactivos que obtuvieron buenos resultados en el procedimiento anterior a un análisis factorial de componentes principales con normalización de Kaiser y rotación ortogonal VARIMAX. Se obtuvieron dos factores $($ Determinante $=0.034 ; \mathrm{KMO}=0.895$; Prueba de esfericidad de Bartlett, $\chi^{2}=0732.3$, $g l=36, p<0.001$ ), el segundo de los cuales estaba compuesto por un solo reactivo por lo que fue eliminado. Un segundo análisis con los ocho reactivos restantes muestra una estructura unifactorial (Determinante $=0.035 ; \mathrm{KMO}=0.896$; Prueba de esfericidad de Bartlett $\chi^{2}=731.1, g l=28$, $p<0.001)$. La consistencia interna de estos reactivos se calculó con la fórmula alfa de Cronbach (Tabla 1).

\section{TABLA 1}

Estructura factorial y principales indicadores del instrumento de ITESN

\begin{tabular}{rc} 
& TESN \\
\cline { 2 - 2 } Rango & $8-49$ \\
Media & 23.6 \\
Desviación estándar & 9.2 \\
Varianza explicada & $53.68 \%$ \\
$\alpha$ de Cronbach & 0.88
\end{tabular}

\section{Reactivos}

En ocasiones pienso que me preocupo demasiado por lo que las otras personas piensen de mí

Me da miedo que los demás se den cuenta de mis errores

0.775

A menudo me da miedo que la gente note mis defectos

Me da miedo que los demás no me acepten

0.752

A menudo estoy preocupado sobre la impresión que otros se forman de mí

Me preocupa lo que las otras personas piensen de mí aun cuando sé que eso no me afecta

Cuando estoy hablando con alguien me preocupa lo que pueda estar pensando sobre mí

A menudo me preocupa que pueda decir o hacer cosas incorrectas

0.637

Fuente: elaboración propia. 


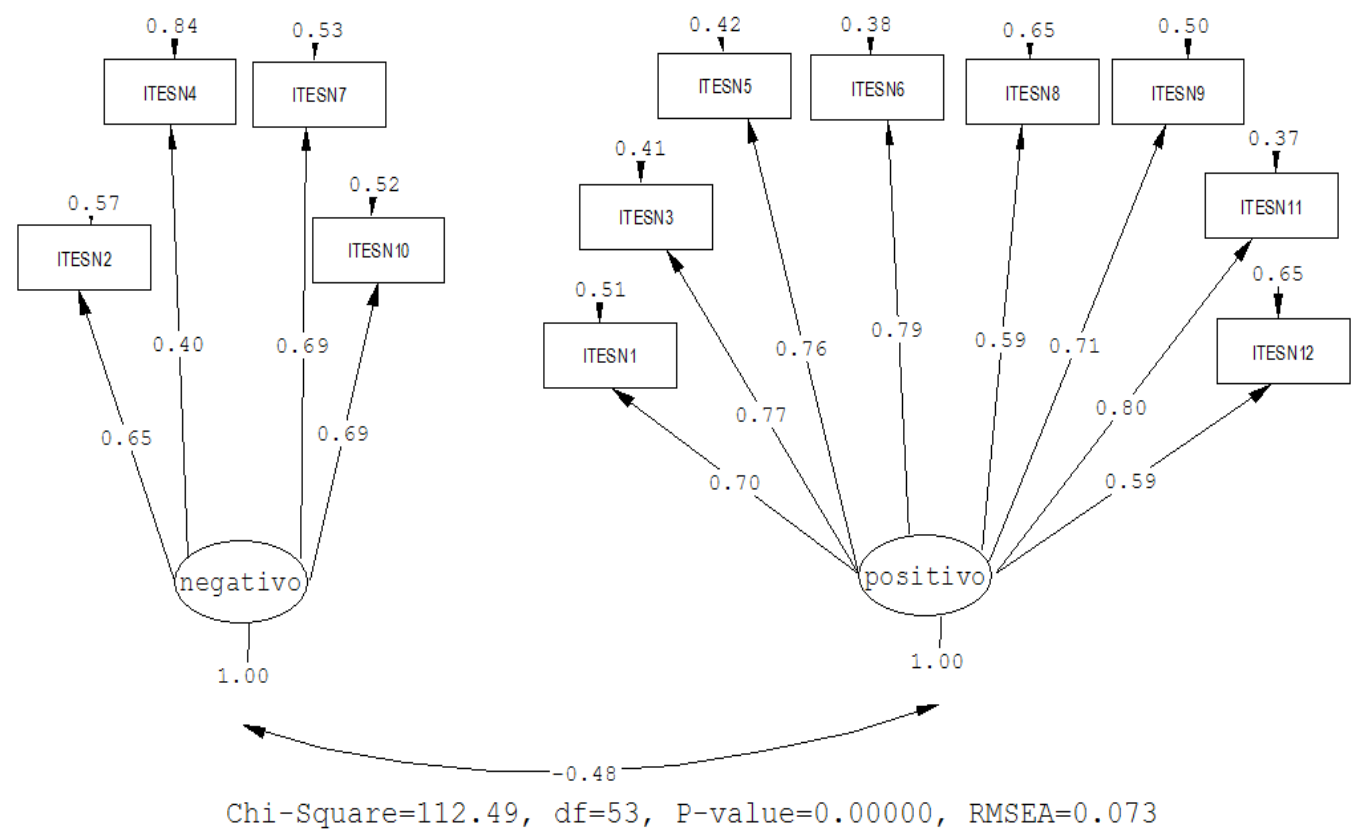

Figura 1. Análisis factorial confirmatorio para el ITESN incluyendo los 12 reactivos.

Fuente: elaboración propia.

\section{Comparación de los modelos de ocho y doce reactivos}

Los análisis factoriales confirmatorios ya citados (Duke et al., 2006; Weeks et al., 2005) mostraron indicadores de bondad de ajuste adecuados para los 12 reactivos del instrumento. Sin embargo, los análisis realizados en este estudio muestran que la mejor estructura para este instrumento estaría compuesta por los ocho reactivos que denotan un alto temor a la evaluación social negativa, dado que los cuatro reactivos redactados negativamente no muestran una buena capacidad de discriminación y representan un estímulo ambiguo para los participantes. Para obtener indicadores que permitan elegir entre la versión completa de 12 reactivos y una de ocho, se realizaron análisis factoriales confirmatorios ${ }^{5}$ mediante el programa LISREL 8.8 (Jöreskog \& Sörbom, 2006).

5 Antes de iniciar este procedimiento se verificó que se cumplieran los siguientes supuestos necesarios para el uso de modelamiento con ecuaciones estructurales: Colinealidad: utilizando regresiones lineales entre cada uno de los reactivos respecto del total, se obtuvieron valores de $r^{2}$ entre 0.559 y 0.745 ; valores
Los resultados para el modelo de dos factores que engloban los 12 reactivos reportado por Duke et al. (2006) muestra un indicador de bondad de ajuste $\chi^{2}$ grande y estadísticamente significativo (Figura 1), reflejando grandes diferencias entre el modelo propuesto y la estructura de la matriz de covarianzas $^{6}$. El indicador Root Mean Square of Asociation (RMSEA) muestra también un ajuste pobre del modelo al ubicarse por encima del valor de 0.05

de tolerancia en todo caso $>0.446$ y de factor de inflación de varianza $<2.24$, descartándose por estos valores un posible problema de colinealidad. Casos atípicos: se buscó a aquellos participantes que reportaran puntajes tres desviaciones estándar por arriba o abajo de la media para descartarlos, pero ninguno fue localizado. Distribución normal: se realizaron pruebas de sesgo y curtosis, obteniendo valores entre 0.115 y 0.759 para el sesgo y entre -0.293 y -1.18 para la curtosis, mostrando en general una distribución semejante a la normal para cada uno de los reactivos. Estos elementos, aunados a la estructura factorial teóricamente encontrada en el análisis factorial previo y al adecuado nivel de consistencia interna observado en el $\alpha$ de Cronbach, fueron considerados como elementos suficientes para el correcto uso del análisis factorial confirmatorio mediante modelamiento con ecuaciones estructurales.

6 Aunque autores como Bentler y Bonnet (1980) proponen que el indicador $\mathrm{c}^{2}$ tiende a sobreestimar las discrepancias entre las covarianzas observadas y el modelo propuesto, aproximaciones más recientes afirman que ese efecto es considerable solo en muestras cercanas a los 5.000 casos (Kline, 2011). 


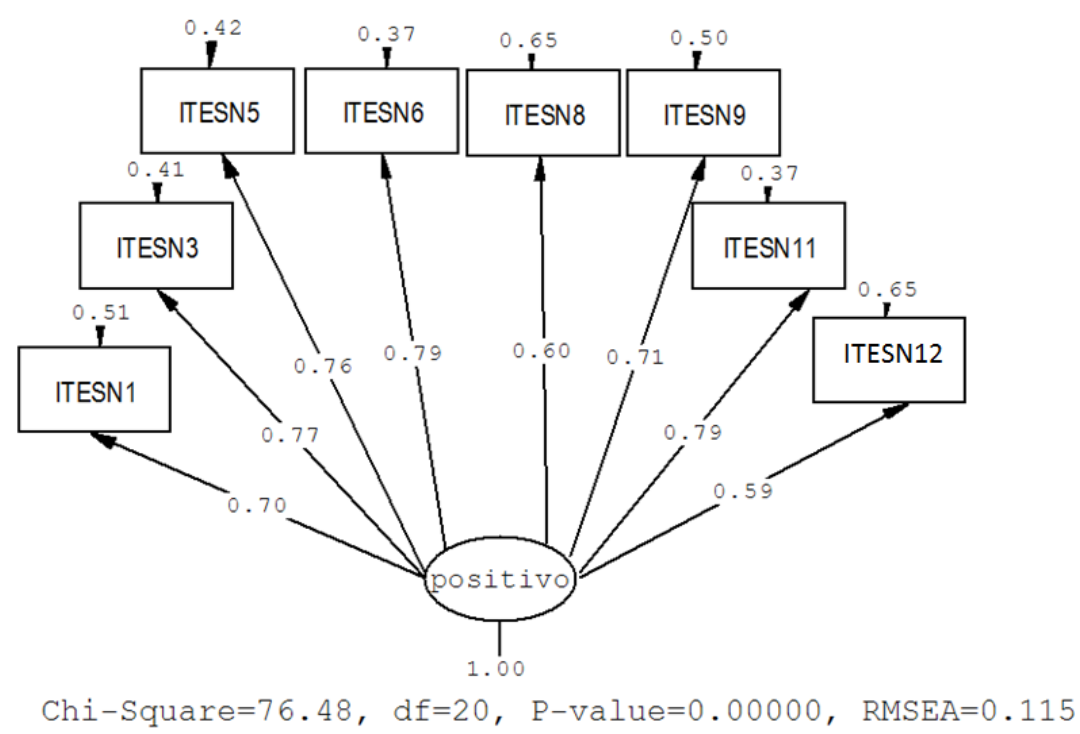

Figura 2. Análisis factorial confirmatorio para el ITESN incluyendo los ocho reactivos redactados positivamente.

Fuente: elaboración propia.

considerado como recomendable tanto en su valor estimado (0.073) como en los intervalos de $90 \%$ de confianza $(0.054 ; 0.091)$. Otros indicadores muestran un mejor ajuste del modelo $(\mathrm{NNFI}=0.97$; $\mathrm{CFI}=0.97$; $\mathrm{AFI}=0.88 ; \mathrm{SRMSR}=0.054) . \mathrm{El}$ error asociado a la medición es, en promedio, más bajo para los reactivos que denotan alto temor a la evaluación social negativa (0.61) que para el otro conjunto de reactivos (0.48). Esta variable latente obtiene además pesos de regresión más altos $(0.71$, en promedio) que la variable que agrupa los reactivos que reflejan bajo temor a la evaluación social negativa. Ambas variables latentes se correlacionaron de forma negativa.

Por su parte, el modelo que integra solo los ocho reactivos redactados afirmativamente en una sola variable latente obtiene un indicador de bondad de ajuste $\chi^{2}$ mucho mejor que el modelo previo, aunque aún refleja diferencias estadísticamente significativas entre el modelo teóricamente propuesto y las covarianzas encontradas en los datos. Por el contrario, el indicador RMSEA refleja un ajuste más pobre que en el modelo anterior tanto en su estimación (0.115) como en sus intervalos de confianza al $90 \%(0.08 ; 0.14)$. A pesar de esto, otros índices señalan un buen ajuste del modelo (NNFI = 0.95; $\mathrm{CFI}=0.97$; $\mathrm{AGFI}=0.85$; $\mathrm{SRMSR}=0.05$ ).

El patrón de resultados obtenidos con esta técnica muestra un ajuste pobre de ambos modelos, $y$ los valores mixtos de los diferentes indicadores no permiten preferir fácilmente a uno sobre el otro. Sin embargo, se eligió el modelo de ocho reactivos dado que los cuatro reactivos redactados negativamente reportan tanto pesos de regresión menores hacia la variable latente como mayores pesos en el error de medición que los ocho reactivos restantes. Esta decisión es congruente con los resultados obtenidos en los análisis de discriminación y factoriales reportados al inicio de esta sección.

\section{Motivación de Auto-Presentación (MAP)}

Como el instrumento de medición de MAP fue diseñado ex profeso para este proyecto, fue necesario contar con indicadores de su funcionamiento psicométrico antes de utilizarlo como referente para los análisis de validez de criterio del ITESN. Para esto, se obtuvieron indicadores de validez y confiabilidad de este instrumento siguiendo el mismo procedimiento utilizado en el ITESN. Se generó una 
TABLA 2

Estructura factorial y principales indicadores del instrumento de MAP

Reactivos

\begin{tabular}{|c|c|c|c|}
\hline & & $\begin{array}{r}\text { Motivaci } \\
\text { Preser }\end{array}$ & $\begin{array}{l}\text { n de auto } \\
\text { tación }\end{array}$ \\
\hline & & Positiva & Negativa \\
\hline & Rango & $3-20$ & $3-20$ \\
\hline & Media & 11.4 & 12.0 \\
\hline Desviación & estándar & 3.7 & 4.6 \\
\hline Varianza e & xplicada & $34.5 \%$ & $32.2 \%$ \\
\hline$\alpha$ de $\mathrm{C}$ & ronbach & 0.77 & 0.72 \\
\hline Reactivos & & & \\
\hline Es importante para mí generar una buena impresión en la persona que voy a conocer & & 0.850 & \\
\hline Me sentiría mal si la persona que voy a conocer se forma una mala impresión de mí & & 0.820 & \\
\hline Voy a tratar de que la persona que voy a conocer se forme una buena impresión de mí & & 0.817 & \\
\hline Si la persona que voy a conocer me rechaza no será algo grave para mí & & & 0.859 \\
\hline Si no le agrado a la persona que voy a conocer no pienso hacer nada para cambiarlo & & & 0.848 \\
\hline No me preocupa lo que la persona que voy a conocer piense de mí & & & 0.687 \\
\hline
\end{tabular}

Fuente: elaboración propia.

variable que sumara los puntajes de los seis reactivos de la escala y se formaron grupos de puntajes altos y bajos con base en los percentiles 25 y 75 . Después, se puso a prueba la capacidad de discriminación de cada reactivo con base al total de la escala utilizando pruebas $t$ de Student ${ }^{7}$ para comparar los promedios de cada reactivo para los grupos con puntajes altos y bajos. Los seis reactivos mostraron discriminar adecuadamente a quienes reportaban puntajes altos o bajos sobre el total de la escala (todas las $t>$ $8.4, p<0.001)$. Posteriormente, fueron sometidos a un análisis factorial de componentes principales con normalización de Kaiser y rotación ortogonal VARIMAX (Tabla 2) (Determinante $=0.220$; $\mathrm{KMO}=0.670$; Prueba de esfericidad de Bartlett $\left.c^{2}=313.3, g l=15, p<0.001\right)$.

7 Los valores de la prueba de sesgo (entre -0.11 y 0.55 ) y curtosis $(-0.25$ y -1.4$)$ reflejan en general una distribución cercana a la normal para todos los reactivos. La prueba de Levene para igualdad de varianzas muestra varianzas equivalentes entre los grupos de puntajes altos y bajos en cuatro de los seis reactivos. En los reactivos en donde no se puede asumir igualdad de varianzas, se utilizaron para el análisis los indicadores ajustados que para este fin provee el paquete estadístico SPSS. Estas características de los reactivos y procedimientos de análisis, aunados al nivel de medición de los reactivos y la independencia de los puntajes entre ambos grupos permiten suponer que se cumplen los supuestos requeridos para el uso de la prueba $t$.

\section{Validez de criterio}

En congruencia con resultados obtenidos previamente (Duke et al., 2006), las mujeres obtienen promedios más altos (24.7) que los hombres (22.4), aunque estas diferencias son apenas marginalmente significativas $t(225)=-1.928 ; p=0.055$.

Otros indicadores de validez de criterio se obtuvieron utilizando correlaciones de Pearson entre el ITESN y el IDAR. Se creó una variable, sumando los puntajes del IDAR, invirtiendo previamente los puntajes obtenidos de los reactivos redactados en un sentido negativo. Altos puntajes en esta variable representaban altos niveles de ansiedad rasgo. Como se esperaba, el ITESN se relaciona positivamente con este indicador $(r=0.563, p<0.001)$.

Otra evidencia de validez de criterio se obtuvo en el contexto del procedimiento experimental de este estudio. Si el ITESN es realmente capaz de medir los niveles de esta variable en los participantes de este estudio, las diferencias en TESN de la medición previa al estímulo experimental (la afirmación de que conocerían a otra persona) deberían generar contrastes en los niveles de ansiedad estado (medidos con el IDAE) ante el escenario de conocer a otra persona. Estos efectos deberían ser 


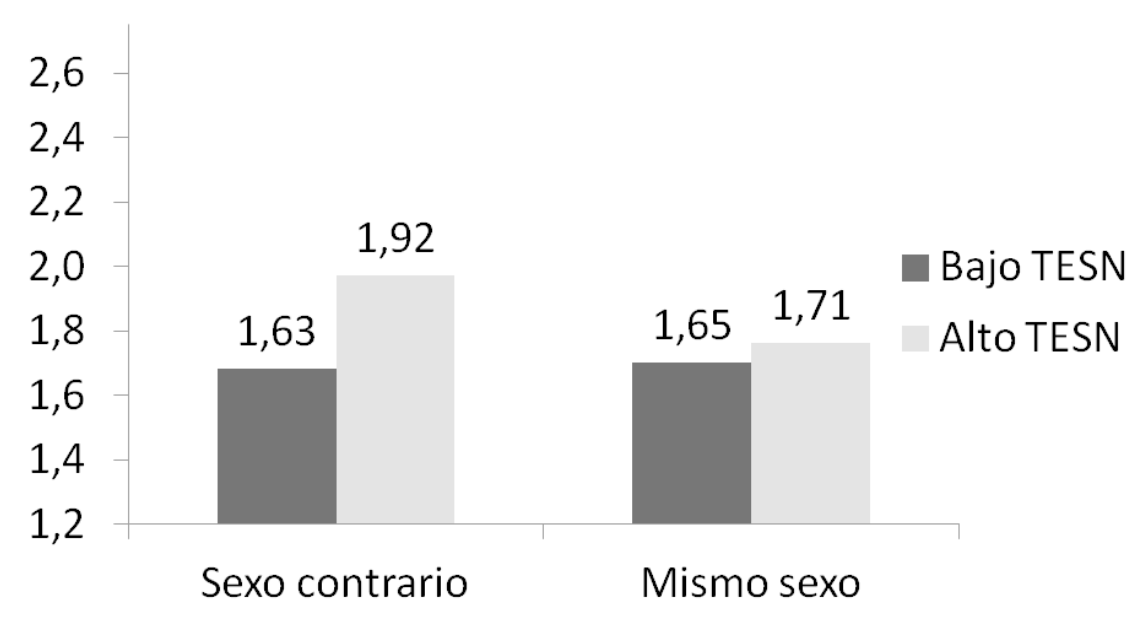

Figura 3. Análisis de varianza factorial comparando la ansiedad estado producida por la manipulación experimental para diferentes niveles de TESN.

Fuente: elaboración propia.

más evidentes cuando la persona que se va a conocer es del sexo opuesto.

Para poner a prueba esta hipótesis se clasificó a los participantes según obtuvieran puntajes altos o bajos en el ITESN, tomando como criterio los percentiles 25 y 75 . Los resultados de un análisis de varianza factorial 2(TESN alto/bajo) X 2 (Interacción con el mismo sexo/sexo opuesto) muestra evidencias que confirman un funcionamiento adecuado del ITESN (Figura 3). El análisis de efectos principales muestra promedios de ansiedad estado más altos en quienes reportaron en un primer momento puntajes altos de TESN (1.84) que en quienes reportaron puntajes bajos (1.64) $F=11.15$, $g l=1.3, p=0.001$. El análisis de interacciones muestra que estas diferencias son especialmente notables en aquellos participantes que conocerán a alguien del sexo opuesto en contraste con quienes conocerán a alguien de su propio sexo $F=4.76$, $g l=1.3, p=0.031$.

Respecto a la medición de MAP, el ITESN se relaciona positivamente con los reactivos que denotan mayor motivación de autopresentación $(r=0.379$, $p<0.001)$. Sin embargo, en contra de lo esperado, la relación entre el ITESN y los reactivos de la medición de MAP que describen baja motivación de autopresentación no fue estadísticamente significativa $(r=0.112, p=0.105)$.

\section{Discusión}

Los análisis de capacidad de discriminación muestran propiedades psicométricas inadecuadas de los reactivos redactados de forma negativa. Se plantea a este respecto dos posibles explicaciones. Una primera posibilidad es que tengan diferentes significados para los participantes. La segunda explicación posible es que la redacción negativa, en combinación con el formato de respuesta, genera reactivos que implican responder a una idea que constituye una doble negación. Este último supuesto ya había sido propuesto por Duke et al. (2006), el cual, sin embargo, sigue requiriendo de prueba empírica que deberá explorarse en posteriores investigaciones.

El pobre desempeño de los reactivos redactados de forma negativa es congruente con estudios previos que han puesto a prueba la validez de esta escala (Duke et al., 2006; Rodebaugh et al., 2004). Aunque estos referentes estaban disponibles al inicio de este proyecto era necesario, considerando posibles diferencias culturales entre las muestras de los diferentes estudios, poner a prueba estas posibilidades antes que darlas por hecho.

Estos resultados y el análisis factorial confirmatorio sugieren que la mejor estructura posible es la compuesta por los ocho reactivos redactados de forma positiva que subyacen a un solo factor. Este 
instrumento obtiene niveles altos de consistencia interna y un patrón de relaciones con otras variables y constructos teóricos que dan cuenta de su validez de criterio: se asocia positivamente con los niveles de ansiedad medida como una diferencia individual; se asocia positivamente con los niveles de ansiedad, medido como un estado emocional, ante la expectativa de interactuar con otra persona y se asocia positivamente con la motivación de autopresentarse ante una persona desconocida a quien se va a conocer. Sin embargo, es necesario considerar los resultados del análisis factorial confirmatorio que muestran indicadores de un pobre ajuste de este modelo.

Este hecho puede dar cuenta de la estructura de dos factores reportada en estudios previos (Duke et al., 2006; Rodebaugh et al., 2004) en lugar de una estructura unifactorial como teóricamente se esperaría. Los pesos beta obtenidos por estos reactivos en el análisis factorial confirmatorio también muestran un desempeño más pobre que los reactivos redactados positivamente.

Las relaciones entre el ITESN y otras variables teóricas son congruentes con lo esperado teóricamente y con lo reportado en estudios previos (Carter et al., 2009; Schlenker \& Leary, 1982). Sin embargo, es necesario explicar la ausencia de relación con el factor de MAP que agrupa los reactivos redactados a la inversa. La solución de dos factores del instrumento de MAP implica que no existe un factor común que subyace a los seis reactivos, reflejándose en patrones de respuesta diferentes para cada factor, aunque no inversos. Es posible que los tres reactivos redactados de forma positiva sean la mejor medición de MAP, mientras que los reactivos redactados de forma negativa generen una interpretación conceptual distinta en los participantes al presentar ideas que se presentan en el formato de una doble negación.

Las correlaciones que dan pie a la validez de criterio fueron más altas para la medición de rasgo (en este caso ansiedad como rasgo) que para las mediciones de estado (ansiedad y MAP ante la expectativa de interactuar con un desconocido). Este resultado es teóricamente congruente, dado que se espera que el temor a la evaluación social negativa sea una característica psicológica que se mantiene estable en los individuos a lo largo del tiempo y a través de diferentes circunstancias. Estas variaciones circunstanciales obtienen también evidencia empírica mediante el procedimiento experimental en este estudio, mostrándose que la expectativa de interactuar con un desconocido incrementa los niveles de ansiedad y motivación de autopresentación y que estos incrementos se asocian con los niveles de temor a la evaluación social negativa de los individuos. Estas evidencias, obtenidas por primera vez en el contexto de un procedimiento experimental, complementan los resultados reportados por Gallego (2010) y Gallego et al. (2007), en lo que respecta a muestras de habla hispana.

Siguiendo la línea de otras investigaciones es importante conseguir evidencias adicionales de su funcionamiento en muestras con un diagnóstico de fobia o ansiedad social y en muestras que no sean de estudiantes universitarios. Sin embargo, se debe considerar que incluso niveles de temor a la evaluación social negativa, que no ameritan un diagnóstico de ansiedad o fobia social, pueden impactar las relaciones sociales y calidad de vida de quienes lo padecen. Desde esta reflexión, se propone seguir investigando el funcionamiento de este instrumento tanto con miras al diagnóstico clínico como para investigar el papel del temor a la evaluación social negativa en la vida cotidiana de la población en general y encontrar alternativas para su manejo saludable.

\section{Referencias}

Ajzen, I. (1991). The theory of planned behavior. Organizational Behavior and Human Decision Processes, 50(2), 179-211.

American Psychiatric Association. (2000). Diagnostic and statistical manual of mental disorders (4a. ed., ed. rev.). Washington, DC: Autor.

Bados, A. (2009). Fobia social. Barcelona: Publicaciones de la Universidad de Barcelona.

Beidel, D. C. \& Turner, S. M. (1998). Shy children, phobic adults. Nature and treatment of social phobia. Washington, DC: APA. 
Bentler, P. M. \& Bonett, D. G. (1980). Significance tests and goodness-of-fit in the analysis of covariance structures. Psychological Bulletin, 88(3), 588-606.

Botella, C., Baños, R. M. \& Perpiñá, C. (2003). Fobia social: avances en la psicopatología, la evaluación y el tratamiento psicológico del Trastorno de Ansiedad Social. Barcelona: Paidós.

Brown, M. A. \& Stopa, L. (2008). The looming maladaptive style in social anxiety. Behavior Therapy, 39(1), 57-64.

Bunnk, B. \& Mussweiler, T. (2001). New directions in social comparison research. European Journal of Social Psychology, 31(5), 467-475.

Carter, M. M., Sbrocco, T. \& Ayati, F. (2009). Predicting anxious response to a social challenge and hyperventilation: Comparison of the ASI and ASI3. Journal of Behavior Therapy and Experimental Psychiatry, 40(3), 434-442.

Díaz-Loving, R. (2002). A bio-psychosocio-cultural approach to couple relationships. En C. von Hofsten \& L. Bäckman (Eds.), Psychology at the turn of the millennium: Social, developmental and clinical perspectives (Vol. 2, pp. 361-392). Brighton, UK: Taylor \& Francis.

Díaz-Loving, R. \& Draguns, J. (1999). Socioculture. Meaning and personality in Mexico and in the United States. En Y. T. Lee, C. McCauley \& J. Draguns (Eds.), Personality and person perception across cultures (pp. 103-126). New Jersey/London: Lawrence Erlbaum Associates Publishers.

Duke, D., Krishnan, M., Faith, M. \& Storch, E. A. (2006). The psychometric properties of the Brief Fear of Negative Evaluation Scale. Journal of Anxiety Disorders, 20(6), 807-817.

Festinger, L. (1954). A theory of social comparison processes. Human Relations, 7(2), 117-140.

Gallego, M. J., Botella, C., Quero, S., Baños R. M. \& García-Palacios, A. (2007). Propiedades psicométricas de la Escala de Miedo a la Evaluación Negativa versión breve (BFNE) en muestra clínica. Revista de Psicopatología y Psicología Clínica, 12(3), 163-176.

Gallego, M. J. (2010). Brief version of the Fear of Negative Evaluation Scale Straightforward Items (BFNE-S): Psychometric properties in a Spanish population. The Spanish Journal of Psychology, 13(2), 981-989.
García, J. (2000). Un estudio de la eficacia entre tres modalidades de tratamiento para población adolescente con fobia social. Tesis doctoral, Universidad de Murcia, Murcia, España. Disponible en http:// www.tdr.cesca.es/TESIS_UM /AVAILABLE/ TDR-0927107-095515//GarciaLopez.pdf

Gartrell, D. (1987). Network approaches to social evaluation. Annual Review of Sociology, 13, 49-66.

Gómez-Jacinto, L. (2005). Comparación social y autoevaluación desde un enfoque evolucionista. Escritos de Psicología, 7, 2-14.

Hernández, G. G., Orellana, V. G., Kimelman, J. M., Núñez, M. C. \& Ibáñez, H. C. (2005). Trastornos de ansiedad en pacientes hospitalizados en medicina interna. Revista Médica de Chile, 133(8), 895-902.

Hidalgo, G. \& Abarca, M. (1990). Desarrollo de habilidades sociales en estudiantes universitarios. Revista Latinoamericana de Psicología, 22(2), 266-280.

Inderbitzen, H. M., Walters, K. S. \& Bukowski, A. L. (1997). The role of social anxiety in adolescent peer relations: Differences among sociometric status groups and rejected subgroups. Journal of Clinical Child Psychology, 26(4), 338-348.

Jöreskog, K. G. \& Sörbom, D. (2006). LISREL 8.8 for Windows. Lincolnwood, IL: Sientific Software International, Inc.

Kline, R. B. (2011). Principles and practice of structural equation modeling (3a. ed.). New York: The Guilford Press.

La Greca, A. M. \& López, N. (1998). Social anxiety among adolescents: Linkages with peer relations and friendships. Journal of Abnormal Child Psychology, 26(2), 83-94.

Leary, M. (1983). A brief version of the Fear of Negative Evaluation Scale. Personality and Social Psychology Bulletin, 9(3), 371-375.

Patterson, C., Kupersmidt, J. \& Griesler, P. C. (1990). Children's perceptions of self and of relationships with others as a function of sociometric status. Child Development, 61(5), 1335-1349.

Rapee, R. M. (1995). Descriptive psychopathology of social phobia. En R. G. Heimberg, M. R. Liebowitz, D. A. Hope \& F. R. Scheneier (Eds.), Social phobia: Diagnosis assessment and treatment (pp. 41-66). New York: Guilford Press. 
Rapee, R. \& Herimberg, R. (1997). A cognitive-behavioral model of anxiety in social phobia. Behaviour Research and Therapy, 35(8), 741-756.

Reyes Lagunes, I. (1993). Las redes semánticas naturales, su conceptualización y su utilización en la construcción de instrumentos. Revista de Psicología Social y Personalidad, 9(1), 81-97.

Rodebaugh, T. L., Woods, C. M., Thissen, D. M., Heimberg, R. G., Chambless, D. L. \& Rapee, R. M. (2004). More information from fewer questions: The factor structure and item proprieties of the original and Brief Fear of Negative Evaluation Scale. Psychological Assessment, 16(2), 169-181.

Sánchez, A. R. \& Díaz-Loving, R. (2009). Reglas y preceptos culturales de la expresión emocional en México: su medición. Universitas Psychologica, 8(3), 793-805.

Schlenker, B. \& Leary, M. (1982). Social anxiety and self-presentation: A conceptualization model. Psychological Bulletin, 92(3), 641-669.

Spielberger, C. D. \& Díaz Guerrero, R. (1975). IDARE. Inventario de ansiedad rasgo-estado. Manual e instructivo. México: El Manual Moderno.

Tavoli, A., Melyani, M., Bakhtiari, M., Ghaedi, H. G. \& Montazeri, A. (2009). The Brief Fear of Negative Evaluation Scale (BFNE): Translation and validation study of the Iranian version. BMC Psychiatry, 9(42), 371-375.
Triandis, H. C. (1977). Interpersonal behavior. Monterey, CA: Brooks/Cole.

Weeks, W. J., Heimberg, G. R., Fresco, M. D., Hart, A. T. \& Turk, L. C. (2005). Empirical validation and psychometric evaluation of the Brief Fear of Negative Evaluation Scale in patients with Social Anxiety Disorder. Psychological Assessment, 17(2), 179-190.

Zacarías, M. (2009). Autoestima y estilos de amor en la elección de pareja. Tesis de licenciatura, Universidad Nacional Autónoma de México, México. Disponible en http://132.248.9.9:8080/tesdig2/ Procesados_tesis_2009/abril/0642219/Index.html

Zubeidat, I. (2005). Evaluación de las características de la ansiedad social específica y generalizada en jóvenes. Tesis de Doctorado, Facultad de Psicología, Universidad de Granada, España.

Zubeidat, I., Fernández Parra, A. \& Sierra, J. (2006). Ansiedad y fobia social: revisión de los autoinformes más utilizados en población adulta e infanto-juvenil. Terapia Psicológica, 24(1), 71-86.

Zubeidat, I., Salinas, J. \& Sierra, J. (2007). Escala de Miedo a la Evaluación Negativa y Escala de Evitación y Malestar Social: fiabilidad y validez en una muestra de adolescentes españoles. Clínica y Salud, 18(1), 67-81. 


\section{Apéndice}

\section{Instrucciones}

Lee cada una de las siguientes frases y marca en el espacio correspondiente qué tanto te describe cada una de ellas. Mientras más grande es el cuadro significa que esa frase te describe Mucho y mientras más chico significa que te describe Poco.

¿Qué tanto te describe cada una de las frases a continuación?

Poco Mucho

1. Me preocupa lo que otras personas piensen de mí aun cuando sé que eso no me afecta

2. NO me preocupa que la gente tenga una mala impresión de mi persona

3. A menudo me da miedo que la gente note mis defectos

4. Rara vez me preocupo sobre qué impresión estoy dejando en alguien

5. Me da miedo que los demás no me acepten

6. Me da miedo que los demás se den cuenta de mis errores

7. NO me molestan las opiniones que otras personas tengan de mí

8. Cuando estoy hablando con alguien me preocupa lo que pueda estar pensando sobre mí

9. A menudo estoy preocupado sobre la impresión que otros se forman de mí

10. El saber que alguien me está juzgando NO tiene ningún efecto en mí

11. En ocasiones pienso que me preocupo demasiado por lo que las otras personas piensen de mí

12. A menudo me preocupa que pueda decir o hacer cosas incorrectas 
